\title{
C15j
}

\section{TEXTURE CLUSTERING OF SATELLITE IMAGES USING SELF-ORGANIZING NEURAL NETWORK}

\author{
Lukashevich M.M. ${ }^{1)}$, Sadykhov R.Kh. ${ }^{2)}$ \\ 1) BSUIR, Minsk, P.Brovka str., 6, Minsk, Belarus kafevm@bsuir.by \\ 2) United Institute of Informatics Problems, Surganov str. 6, Minsk, Belarus rsadykhov@bsuir.by
}

\begin{abstract}
The goal of this paper is to present a texture clustering system for remote sensing image data. Texture information is useful for image data browsing and retrieval. Authors present the results of self-organizing neural network design for solving the clustering task of gray scale remote sensing image data. The architecture of neural network and the learning algorithms for this network such as: algorithm WTA (Winner Takes All), algorithm CWTA (Winner Takes All with Conscience) and classic Kohonen algorithm WTM (Winner Takes Most - the Winner receives more) are considered. Some experimental results using textures of the Brodatz album, multi-spectral and radar images are also represented.
\end{abstract}

Keywords: self-organizing neural network, clustering, texture, remote sensing data

\section{INTRODUCTION}

In the recent time artificial neural networks (NN) have found wide application in analysis and image recognition problems.

In the conditions of increasing the data received by systems of remote sensing the $\mathrm{NN}$ are the main tool to develop the methods and algorithms for remote sensing data processing. The application of NN allows to extend possibilities of traditional methods for data analysis in geoinformation systems (GIS), and to create more powerful tools for remote sensing image data processing $[1,2]$.

GIS is a good environment and tool for implementation of artificial intelligence methods and expert systems. The analysis of closeness for selected classes between itself and with other objects is necessary for interpretation of satellite information. In practice the different algorithms of factor and cross-correlation analysis are used for this task. The usage of these methods is an ineffective for large data volumes, and especially for initial data with nonlinear character of interrelations.

The NN algorithms are good alternative for solving this problem. This is a generalization of linear statistic methods for nonlinear case.

Neural network is one the most powerful tools for solution of poorly formalized tasks. The wide distribution of neural networks is explained the following merits of neural network approach:

1. Automatic adjustment of neural model parameters for a task decision. Human intervention doesn't require for model construction which solving the task.

2. Universality. Neural network allows solving any tasks by standard way without taking semantics into consideration. Such tasks can be presented like examples.

3. Steady state under working with noisy or doubtful data.

4. Possibility for adaptation to new conditions.

5. Fault resiliency and stability to data corruption.

6. High parallelism inherent to neural models [35].

\section{THE GENERAL THEORETICAL DATA}

The issues of clustering or groupings of objects are considered in many fields of science. The term "clustering" is usually understood as grouping of objects by similarity of their description, and the term "cluster" is a group of objects that are similar among themselves $[6,7]$.

In artificial intelligence the clustering problem is solved with the use of self-organizing NN. There are two basic approaches among the self-organizing mechanisms: the self-organization based on associative Hebb rule $[8,9]$ and the competitive mechanism between neurons based on Kohonen rule [10-13].

The area of artificial $\mathrm{NN}$ is dynamic and quickly developing. The NN approach has been used in wide spread of applications in various fields of a science, 
including the analysis and image recognition area $[14,15]$.

\section{FEATURE SELECTION FOR CLUSTERING}

The problem of image clustering is closely connected with the question of signatures or informative features extraction from initial data. Clusters are image elements (set of pixels), that are similar on brightness, texture and etc.

The simplest widespread practice is an elementwise (pixel) classification of satellite images with the use of their spectral brightness. However it often gives incorrect decision. The majority of classes which are presented in satellite images have high variability of reflection characteristics. Therefore the creation of more effective methods for the analysis and recognition of satellite images is very important when the problem of contextual information is growing. This information can be taken from statistical coherences of the neighboring elements of an image and a spatial data structure. Therefore exploiting texture analysis methods of images is a more promising way.

In the presented task of the textural analysis it is necessary to allocate following points (fig. 1):

1. Choice the system of textural attributes.

2. Choice by an expert the NN architecture.

3. NN learning by texture samples.

4. Image clustering.

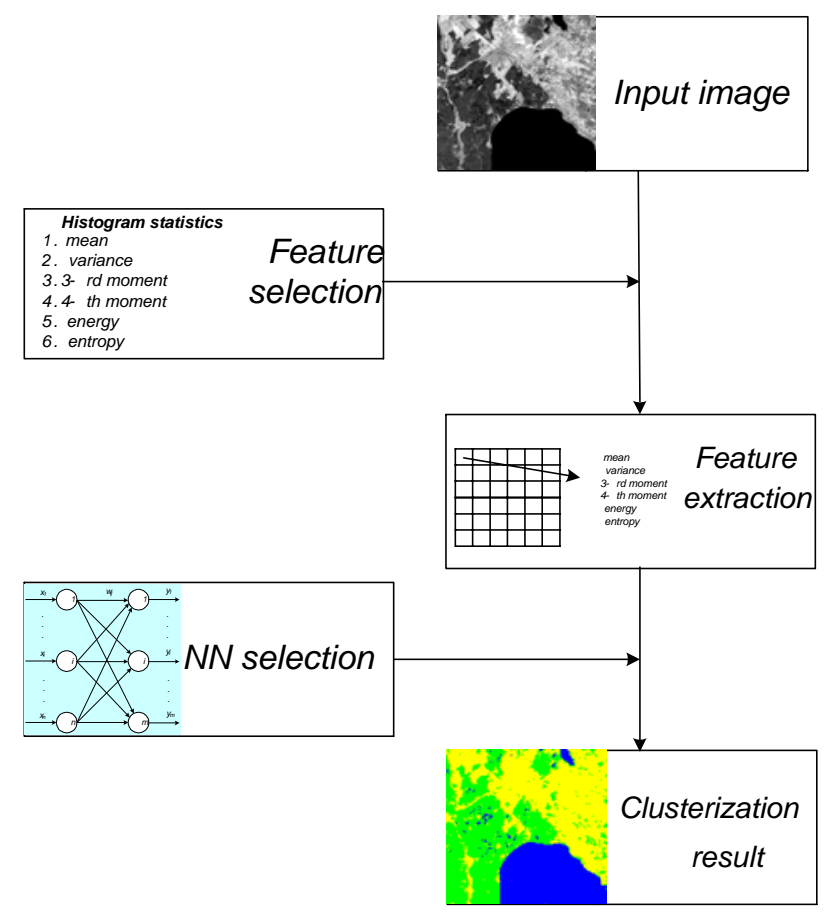

Fig. 1 - Algorithm the texture clustering for satellite images

The easiest approach for describing the texture is the using statistical moments of the grey-level histogram of an image or region [16-19]. A statistical approach with histogram statistics calculation was used on the stage of input data forming for image clustering.

Let $x=0,1, \ldots, N-1$ is a width of an image, $y=0,1, \ldots, M-1 \quad$ - is an image height, and $i=0,1, \ldots, G-1-$ is a number of image intensity levels.

Then, the number of pixels of the images, that have intensity $i$, is calculated as follows:

$$
h(i)=\sum_{x=0}^{N-1} \sum_{y=0}^{M-1} \delta(f(x, y), i),
$$

where $\delta(j, i)$ is the Kroneker delta function.

The probability of occurrence of pixel with intensity $i$ can be calculated using the relation:

$$
p(i)=\frac{h(i)}{N^{*} M}
$$

If we know the probability for each value of pixel's intensity of the image, it is possible to evaluate the following histogram statistics:

Mean

$$
\mu=\sum_{i=0}^{G-1} i p(i)
$$

Variance

$$
\sigma^{2}=\sum_{i=0}^{G-1}(i-\mu)^{2} p(i)
$$

3-rd moment

$$
\mu_{3}=\sum_{i=0}^{G-1}(i-\mu)^{3} p(i)
$$

4-th moment

$$
\mu_{4}=\sum_{i=0}^{G-1}(i-\mu)^{4} p(i)
$$

Energy

$$
E=\sum_{i=0}^{G-1}[p(i)]^{2}
$$

Entropy 


$$
H=-\sum_{i=0}^{G-1} p(i) \log _{2}[p(i)]
$$

Because variance values tend to be large for grayscale images, it is necessary to normalize the variance to the interval [0 1]

$$
R=1-\frac{1}{1+\sigma^{2}}
$$

The texture features listed above for three types of texture (fig. 2) are presented in table 1.
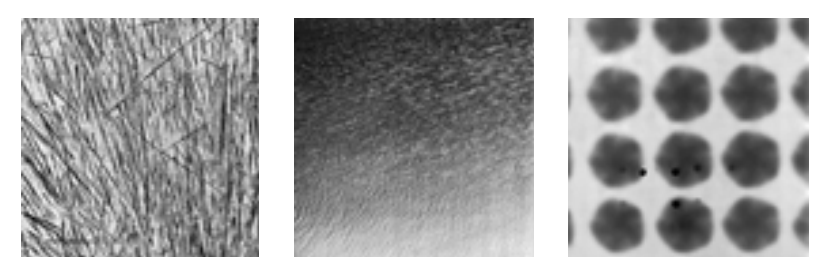

Fig. 2 - Texture examples from Brodatz database

Table 1. Histogram Statistics

\begin{tabular}{|l|l|}
\hline \multicolumn{2}{|c|}{ Histogram statistics } \\
\hline \multicolumn{1}{|c|}{ Image } & \multicolumn{1}{c|}{ D15 } \\
\hline mean & 147,53 \\
\hline variance & 4598,3 \\
\hline 3-rd moment & $-1,3515 \mathrm{e}+005$ \\
\hline 4-th moment & $3,6083 \mathrm{e}+007$ \\
\hline energy & 0,025876 \\
\hline entropy Image & Not-a-Number \\
\hline & \multicolumn{1}{c|}{ D38 } \\
\hline mean & 114,87 \\
\hline variance & 3327,3 \\
\hline 3-rd moment & 43942 \\
\hline 4-th moment & $2,0517 \mathrm{e}+007$ \\
\hline energy & 0,013863 \\
\hline entropy & Not-a-Number \\
\hline & D48 \\
\hline mean & 149,76 \\
\hline variance & 3956,3 \\
\hline 3-rd moment & -43753 \\
\hline 4-th moment & $2,1501 \mathrm{e}+007$ \\
\hline energy & 0,024682 \\
\hline entropy & Not-a-Number \\
\hline
\end{tabular}

\section{SELF-ORGANIZING NN STRUCTURE}

The question of self-organizing NN synthesis was examined within the framework of this article. The self-organizing NN are those that are used for unsupervised learning. Such networks are used for finding the more compact data definition: compression, clustering, features extraction.
In general case the self-organizing $\mathrm{NN}$ is a twolayer NN. The first layer is the input layer which contains $\mathrm{n}$ neurons. It is used for a reception and distribution of vector of texture features $x=\left(x_{1}, x_{2}, \ldots, x_{i}, \ldots, x_{n}\right)$. The neurons number in this layer can change depending on the number of texture features used for clustering.

The second layer contains $m$ neurons defined as the number of clusters.

A vector $w^{j}=\left(w_{1 j}, w_{2 j}, \ldots, w_{i j}, \ldots, w_{n j}\right)$ is the vector of weights coefficients, reflecting connections of neurons from the first layer with the neurons of the second layer. The structure of the self-organizing $\mathrm{NN}$ is presented on a fig. 3 .

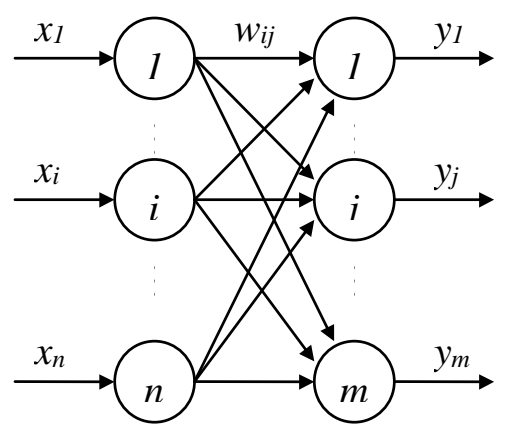

Fig. 3 - Self-organizing NN Structure

\section{NN LEARNING}

\section{A. GENERAL REMARKS}

In the first step the image is divided by blocks which have a size of $3 \times 3$ (fig. 4 ). Half of the blocks is used for NN learning. The texture features are calculated for each of the blocks and in the next step they are used as input vectors for NN learning. Then after NN learning the texture features vectors for all blocks of image are given on the $\mathrm{NN}$ entrances. Array of structures

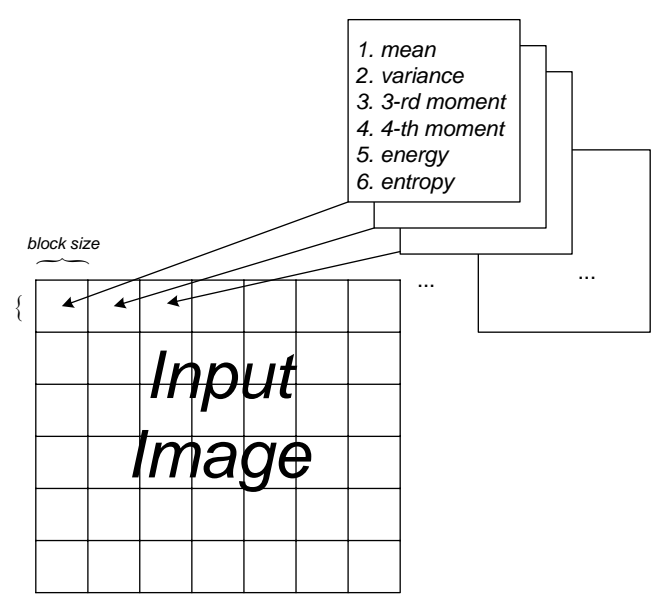

Fig. 4 - Image is divided by blocks 
The following algorithms have been used in experiments for decision problem of clustering of gray level remote sensing image data. These algorithms are based on the competition mechanism between neurons:

- algorithm WTA (Winner Takes All);

- algorithm CWTA (Winner Takes All with Conscience);

- classic Kohonen algorithm WTM (Winner Takes Most).

\section{B. VECTORS NORMALIZATION}

Performing the vectors normalization for $x$ and $w$ is needed for correct dividing features space. Scientific researches from some authors have confirmed the necessity of normalization. Especially it is necessary when we use small feature space like in this case.

The following method of vector components resize was chosen from usual methods of normalization and based on application the next relation:

$$
x_{i} \leftarrow \frac{x_{i}}{\sqrt{\sum_{i=1}^{N} x_{i}^{2}}}
$$

\section{WTA, CWTA ALGORITHMS}

Experiments in which the known algorithms WTA and CWTA were used for NN learning showed the following results. The algorithm CWTA gives the best results because it allows us to take into account the conscience factor of neurons. The amount of victories is considered for every neuron. Neurons with less activity are encouraged for chances equality.

In the learning process a neuron-winner is determined at the produce of every input vector on the base of the maximum for value (11):

$$
y_{j}=\sum_{i=1}^{n} w_{i j} x_{i}=\left|w^{j}\right||x| \cos \alpha
$$

If we use normalized vector, then scalar product is equal to Euclidean distance between an input vector and weight vector. Therefore in practice a neuron-winner is determined as minimum product of Euclidean distance between an input vector and weights vector and the number of victories of this neuron.

$$
d_{v}=\min \left(\left|x-w^{j}\right| f_{j}\right)
$$

During the casual initialization of weights coefficients matrix some neurons can appear in space area, where data is not present in general. Initially every neuron gets one victory for equalization of chances for victory and one victory for the removal of "dead neurons" problem.

For a neuron-winner connections increase the next relation has been applied:

$$
w_{i j}(t+1)=w_{i j}(t)+\beta\left(x_{i}-w_{i j}(t)\right)
$$

Learning proceeds until a maximum of Euclidean distance between any input vector and proper weights vector of neuron-winner will not reach a set value. This value was searched experimentally.

\section{CLASSIC KOHONEN ALGORITHM WTM}

Kohonen [5-7] suggested to add the learning information about the location of neurons on the output layer to the rule of competition. For this purpose neurons are put in order on one- or twodimensional maps and then special function is entered. It corrects the change of scales depending on the distance to the neuron-winner. $h(t, k, j)-$ is the force of influencing between a neuron-winner $k$ and a neuron $j$ in the moment of time.

It should be noted that the time the radius of influencing usually is narrowed. If we use this function, the weights are changed for all neurons of network, rather than just for the neuron-winner:

$$
w_{i j}(t+1)=w_{i j}(t)+\beta h(t, k, j)\left(x_{i}-w_{i j}(t)\right)
$$

In experiments Gaussian bell has been used as such function. A parameter $\sigma$ which depends on time was used in the function denominator (fig. 5).

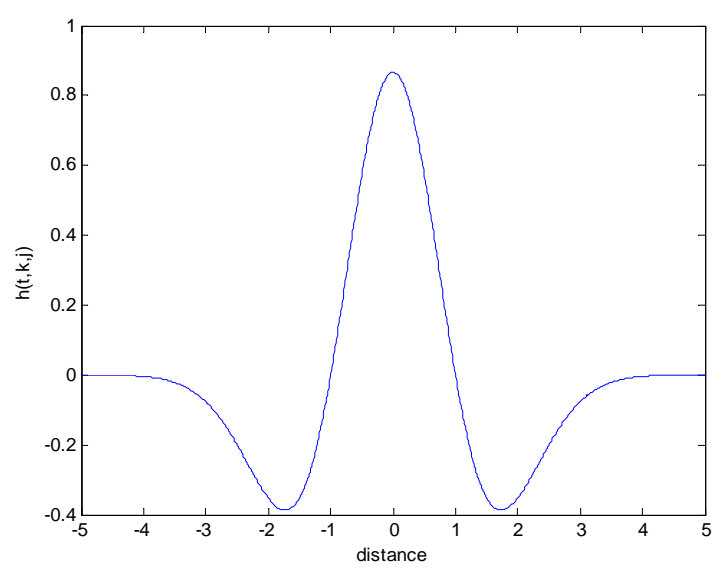

Fig. 5 - Distance function 
The experiments have been performed using texture of the Brodatz, multi-spectral and radar images. They showed that this algorithm gave worst results as against CWTA algorithm.

\section{EXPERIMENTS RESULTS}

Some experimental results using textures of the Brodatz album, multi-spectral images from Landsat $7 \mathrm{ETM}+$ (fig. 6) and radar images from TerraSAR-X (fig. 8) are represented here.

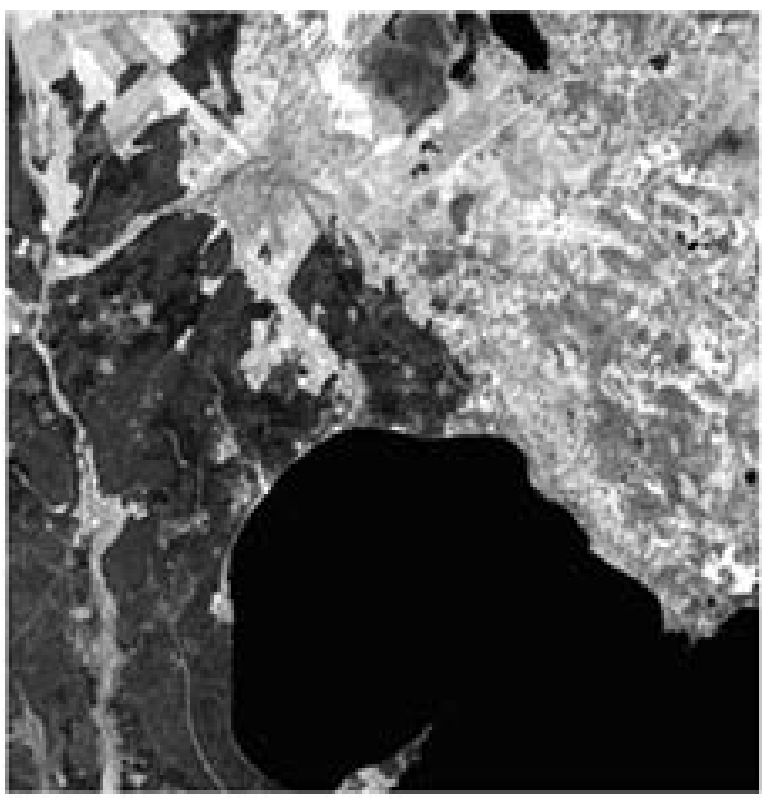

Fig. 6 - Image from Landsat 7 ETM+

The clustering results of satellite infrared image are presented on a fig.7. This multi-spectral image was got from Landsat 7 ETM+.

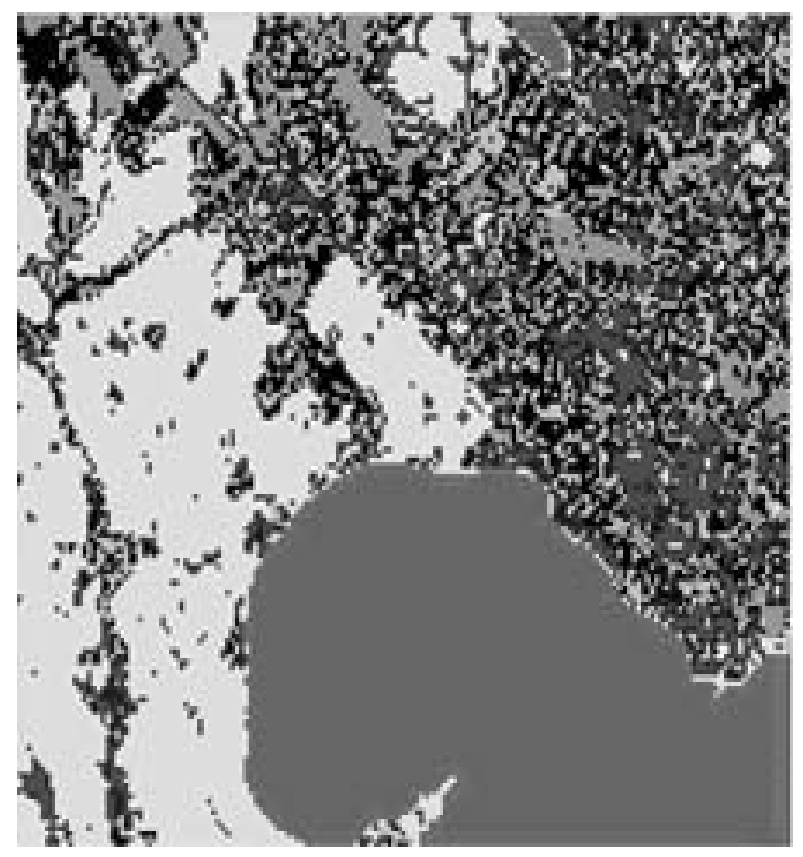

Fig. 7 - Clustering result using the self-organizing NN
The clustering results of SAR image are presented on a fig. 9. This multi-spectral image was got from TerraSAR-X.

The use of self-organizing NN showed that for the decision of clustering problem of gray level remote sensing image the self-organizing $\mathrm{NN}$ gives the same good results as well as classic iterative Kmeans algorithm.

In the synthesis of self-organizing NN CWTA algorithm showed the best results as against WTA algorithm and classic Kohonen algorithm.

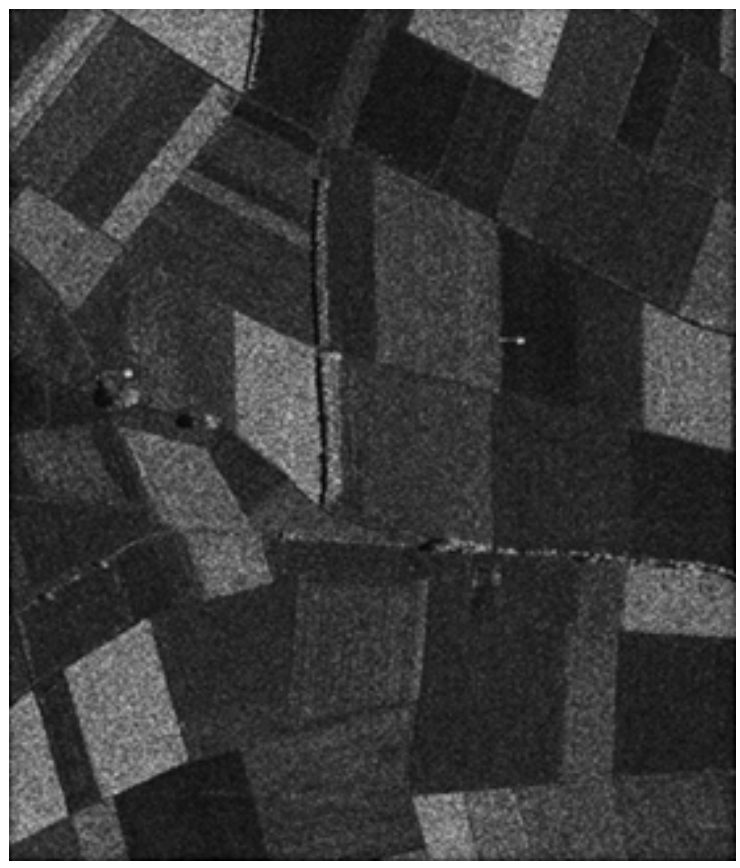

Fig. 8 - Image from TerraSAR-X

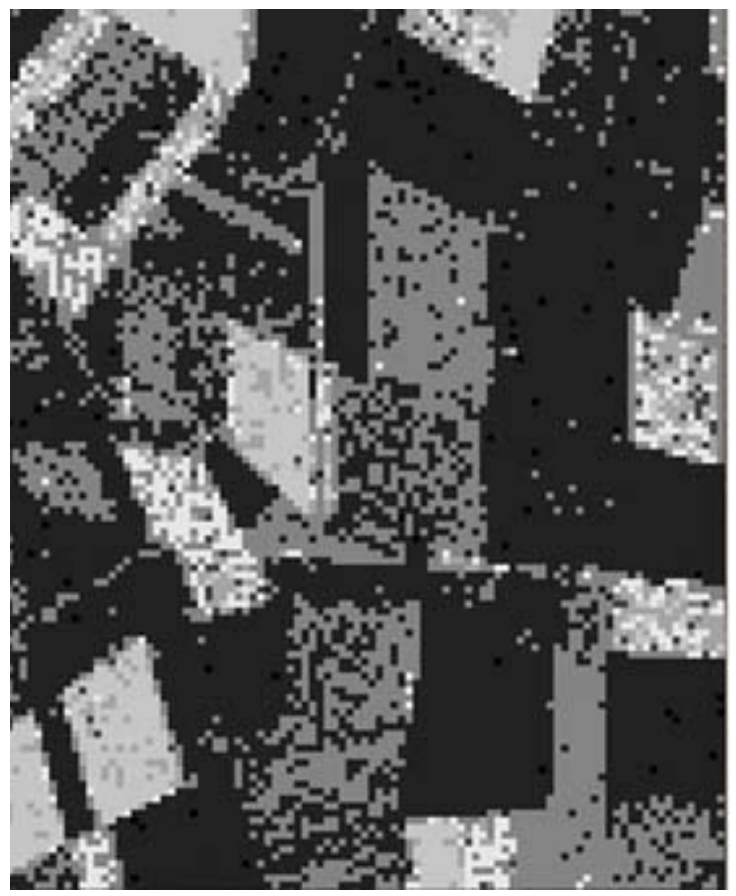

Fig. 9 - Clustering result using the self-organizing NN 
However it is needed to understand that does not exist single universal clustering algorithm. If we use any algorithm it is important to understand its merits and disadvantages, taking into account the nature information.

\section{CONCLUSIONS}

Authors presented the results of self-organizing neural network design for the solving of clustering task of gray scale remote sensing image data. Testing results showed good accuracy of $\mathrm{NN}$ approach for clustering of gray scale satellite image as against to the classic clustering algorithms.

\section{REFERENCES}

[1] M.M. Lukashevich, R.Kh. Sadykhov. Synthesis of a Self-Organizing Neural Network in a Problem of Clustering of Gray Level Remote Sensing Image Data. Proceedings of the Fifth International Conference "Neural Networks and Artificial Intelligence" (ICNNAI'2008), Minsk, Belarus 27-30 May 2008, pp. 306-309.

[2] R.Kh. Sadykhov, M.M. Lukashevich. The Algorithm to Process the Earth Images on the Base of the Energetical Texture Features. (in Russian). Proceedings of the Belarussian Space Congress, Minsk, Belarus 23-25 October 2007, pp. 144-148.

[3] S. Osowski. Neural networks for information processing (in Russian). Finances and statistics. Moscow, 2002. - p. 344.

[4] S. Haykin. Neural Networks: A Comprehensive Foundation (2nd Edition) (in Russian). Publishing house "Vil'yams". Moscow, 2006. p. 1104.

[5] V.A. Golovko. Neural networks: learning, organization and application: train aid for the higher institutes. IPRZHR. Moscow, 2001. p. 256.

[6] J. Ven Rayzin. Classification and cluster (in Russian). Mir. Moscow, 1980. - p. 389.

[7] E. Khant. Artificial intelligence (in Russian). Mir. Moscow, 1978. - p. 558.

[8] D. Hebb. Organization of behavior. J. Wiley. New York, 1949. - p. 335.

[9] J. Hertz, A. Krogh, R. Palmer. Introduction to the theory of neural computation. AddisonWesley. New York, 1991. - p. 327.

[10] T. Kohonen. Self-organization and associative memory. Springer-Verlag. New York, 1989. p. 312.

[11] T. Kohonen. Self-organized formation of topologically correct feature maps, Biol. Cybernetics 43 (1982). p. 56-69.

[12] T. Kohonen. Self-organizing maps (2-nd edition). Springer. Berlin, 1997. - p. 426.
[13] T. Kohonen, Self-Organizing maps $\left(3^{\text {rd }}\right.$ edition), vol. 30. Springer. Berlin, Heidelberg, 2001. - p. 501.

[14] R.Kh. Sadykhov, M.E. Vatkin. Algorithm of gray scale images processing of integral microcircuits based on the "Neokognitron" neural network. Digital image processing. Collected scientific papers, Number 5. Minsk, 2001. - p. 68-75.

[15] A.V. Zamyatin. Using of artificial neural networks for multi-spectral aerospace images classification. Proceedings of the VI all-russian scientific practical conference, Moscow 2004, - p. 239-246.

[16] M.M. Lukashevich, R.Kh. Sadykhov The Algorithm of Texture Segmentation with the Use Energetical Characteristics. (in Russian) BSUIR Reports 6 (36) Minsk (2008). - pp. 109116.

[17] M. Tuceryan, A.K. Jain. Texture Analysis. The handbook of Pattern Recognition and Computer Vision ( $2^{\text {nd }}$ Edition). World Scientific Publishing Co. Singapore, 1998. - p. 207-248.

[18] A. Materka, M. Strzelecki. Texture Analysis Methods - A Review. Technical University of Lodz, Institute of Electronics, COST B11 report. Brussels, 1998. - p. 33.

[19] L.G. Shapiro, G.C. Stockman. Computer Vision(in Russian). BINOM. Moscow, 2006. p. 752.

[20] R. Sadykhov, M. Lukashevich. Texture Segmentation Of Satellite Images By Neural Network Approach. Proceedings of the Second International Conference "Problem of Cybernetics and Informatics (PCI'2008)", Baku, Azerbaijan 10-12 September 2008, - pp. 167-170.

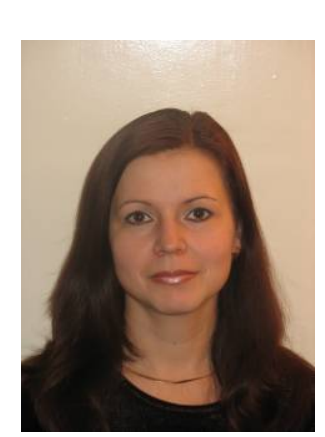

Lukashevich Marina received her BEng. degree in 2005 and M.Sc. degree in 2007 in Computer Systems and Networks from The Belarusian State University of Informatics and Radioelectronics (BSUIR). She is currently an assistant at Department of Computer Systems and Networks and she is a Ph. D. student at the same university.

Her current research interests are artificial neural network, pattern recognition and image processing. 


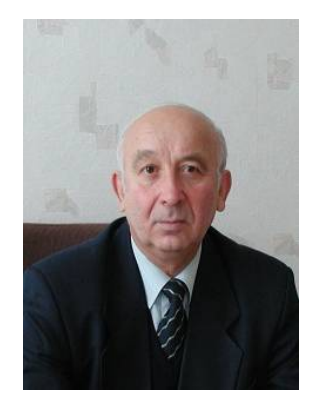

Sadykhov Rauf Kh. He received his BEng. Degree in 1967 from The Azerbaijanian polytechnic institute (Baku). He received his Ph.D. from The Institute of Technical Minsk. In 1991 he received Doctor of science degree.

From 1995 he is a head of Computer Systems department in The Belarusian State University of Informatics and Radioelectronics (BSUIR).

His main research areas includes applications of digital signal and image processing, pattern recognition and identification, computer vision. 